\title{
Auch bei adipösen Jugendlichen ist die „Messerdiät" effektiv - mit gewissen Mankos
}

Fragestellung: Wie steht es um die Wirksamkeit und Sicherheit der bariatrischen Chirurgie bei Jugendlichen mit morbider Adipositas?

Hintergrund: Die bariatrische Chirurgie wird zunehmend für die Behandlung der schweren Adipositas auch bei Jugendlichen erwogen. Allerdings gibt es nur wenige prospektive Studien, die die Wirksamkeit und Sicherheit dessen untersucht haben und damit ein wichtige Hilfe für Indikationsstellungen wären.

Patienten und Methoden: Die Autoren rekrutierten prospektiv aus fünf Zentren in den USA 242 Jugendliche, die sich einer bariatrischen Operation unterzogen. Die analysierten Patienten erhielten entweder einen Roux-en-Y-Magenbypass (161 Teilnehmer) oder einen Schlauchmagen (67 Teilnehmer). Veränderungen von Körpergewicht, Ko-Morbiditäten, kardiometabolischen Risikofaktoren, die gewichtsassoziierte Lebensqualität und post-

\section{Originalie}

Inge TH, Courcoulas AP, Jenkins TM et al. Teen-LABS Consortium. Weight Loss and Health Status 3 Years after Bariatric Surgery in Adolescents. N Engl J Med 2016; 74(2):113-23. operative Komplikationen wurden über einen Zeitraum von drei Jahren erhoben.

Ergebnisse: Das mittlere $( \pm$ SD) Alter der Teilnehmer war 17 $\pm 1,6$ Jahre, der mittlere Body-Mass-Index war 53 $\mathrm{kg} / \mathrm{m}^{2} ; 75 \%$ der Teilnehmer waren weiblich, $72 \%$ hatten eine weiBe Hautfarbe.

3 Jahre nach dem Eingriff hatte sich das mittlere Gewicht um 27\% (95\%-KI, 25-29) in der Gesamtkohorte, um 28\% (95\%-KI, 25-30) bei gastrischem Bypass und um 26\% (95\%-KI, 22-30) in der Schlauchmagengruppe reduziert. 3 Jahre nach dem Eingriff zeigte sich eine Remission des Typ-2-Diabetes bei 95\% (95\%-KI, 85 bis 100) der Teilnehmer, die zu Beginn der Studie einen Diabetes aufwiesen. Die Remission einer abnormalen Nierenfunktion trat bei $86 \%$ auf (95\%-KI, 72-100), die eines Prädiabetes bei 76\% (95\%-KI, 56-97), die eines erhöhten Blutdrucks bei 74\% (95\%-KI, 64-84) und die einer Dyslipidämie bei 66\% (95\%KI, 57-74). Die gewichtsassoziierte Lebensqualität verbesserte sich ebenfalls signifikant.

Dagegen zeigte sich 3 Jahre nach dem Eingriff eine Hypoferritinämie bei 57\% (95\%-KI, 50-65) der Teilnehmer und $13 \%$ (95\%-KI, 50-65) der Teilnehmer hatten sich einer oder mehreren zusätzlichen intraabdominellen Eingriffen unterzogen.

Schlussfolgerungen: In dieser multizentrischen, prospektiven Studie zur bariatrischen Chirurgie bei Jugendlichen zeigten sich nach drei Jahren ein signifikant verringertes Gewicht sowie eine verbesserte kardiometabolische Gesundheit und gewichtsassoziierte Lebensqualität. Risiken, die mit dem chirurgischen Eingriff verbunden sind, waren der Mangel an Mikronährstoffen und zusätzliche abdominelle Eingriffe.

\section{- Kommentar von Prof. Dr. Nanette C. Schloot}

\section{Eisenspiegel im Blick behalten}

Anhand dieser Studie wird deutlich, dass die bariatrische Chirurgie auch bei Adoleszenten mit morbider Adipositas (BMI $>50 \mathrm{~kg} / \mathrm{m}^{2}$ ) zu einer verbesserten Gesundheit führt, auch wenn es in 13\% der Fälle zu zusätzlichen Operationen und in 57\% der Fälle zu einem Eisenmangel kam.

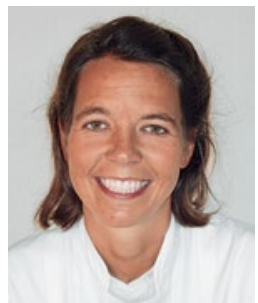

Prof. Dr. med. Nanette C. Schloot

Deutsches Diabetes-Zentrum

Institut für Klinische Diabetologie an der Heinrich-Heine Universität Düsseldorf Nanette.Schloot@DDZ.uni-duesseldorf.de

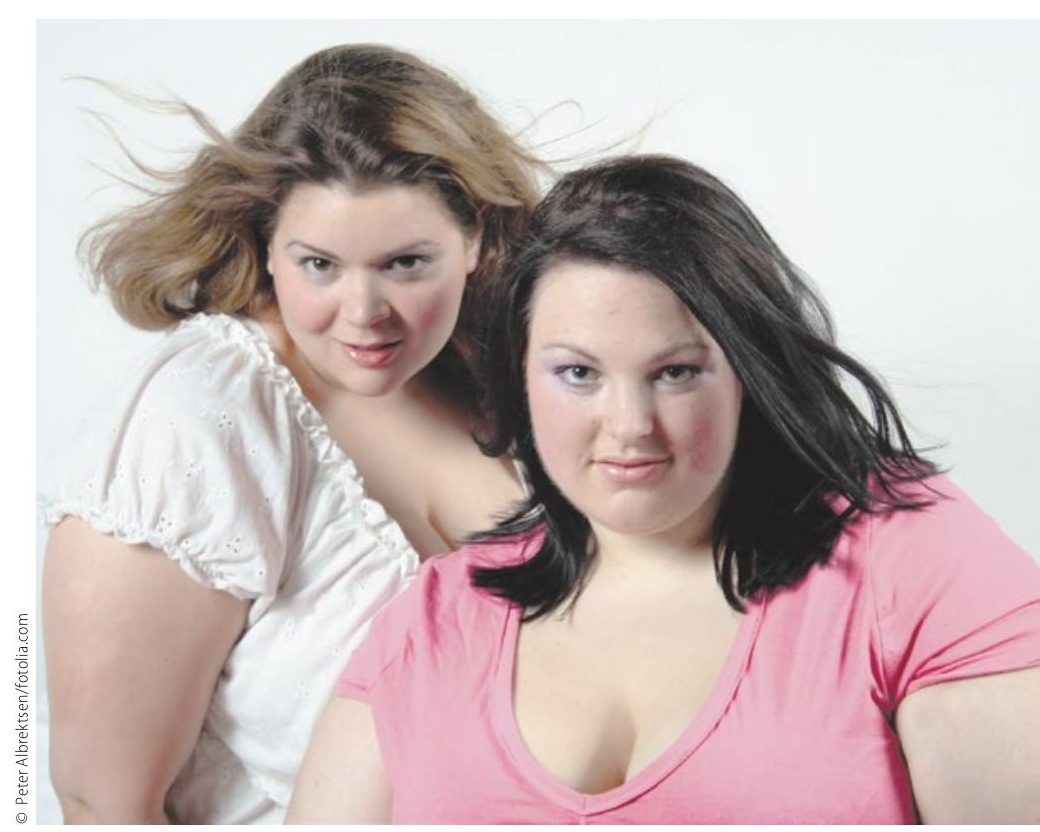

Auch für junge Menschen kann die bariatrische Chirurgie eine Option sein, Für und Wider sollten nur besonders sorgfältig abgewogen werden. 